\title{
Knowledge, attitude and practice of students towards blood donation in Arsi university and Adama science and technology university: a comparative cross sectional study
}

\author{
Habtom Woldeab Gebresilase ${ }^{1}$, Robera Olana Fite ${ }^{2^{*}}$ (D) and Sileshi Garoma Abeya ${ }^{3}$
}

\begin{abstract}
Background: Blood can save millions of lives. Even though people do not donate blood regularly, there is a constant effort to balance the supply and demand of blood. The aim of this study was, therefore, to determine the knowledge, attitude and practice of blood donation between university students.

Methods: The comparative cross sectional study design was used in Adama Science and Technology University and Arsi University from April 11-May 2, 2016.360 students were selected using stratified sampling. Frequencies and proportions were computed. Chi-Square and logistic regressions were carried out and associations were considered significant at $p<0.05$.

Result: The study revealed that there was a significant knowledge difference $(X 2=152.779, p<0.001)$ and Attitude difference ( $X 2=4.142, p=0.042$ ) between Health Science students of Arsi University and Non-Health Science students of Adama Science and Technology University. The gender of the students (AOR =3.150, 95\% Cl: 1.313, 7 . 554) was a significant predictor of the level of knowledge of Health Science students. The ethnicity of students ( $A O R=2.085,95 \% \mathrm{Cl}: 1.025,4.243$ ) was a significant predictor of the level of an attitude of Health Science students and gender of students (AOR $=0.343,95 \% \mathrm{Cl}: 0.151,0.779$ ) was a significant predictor of the level of an attitude of Health Science students. Concerning Non-Health Science students, religion (AOR $=10.173,95 \% \mathrm{Cl}$ : 1.191, 86.905) and original residence $(A O R=0.289,95 \% \mathrm{Cl}: 0.094,0.891)$ were a significant predictor of the level of knowledge of Non-Health Science students. Gender (AOR $=0.389,95 \% \mathrm{Cl}: 0.152,0.992)$ and Year of study (AOR $=0.389(0.164,0$. 922) were significant predictor of level of attitude of Non-Health Science students. Year of study (AOR $=5.159,95 \%$ $\mathrm{Cl}$ : 1.611, 16.525) was a significant predictor of level of practice of Health Science students.
\end{abstract}

Conclusion: Significant knowledge difference and attitude difference were observed between students from Arsi University and Adama Science and Technology University.

Keywords: Knowledge, Practice, Attitude, Health science, Non-health science

\footnotetext{
* Correspondence: rolana2000@gmail.com

${ }^{2}$ Department of Nursing, College of Health Sciences and Medicine, Woliata

Sodo University, PO-Box: 138, Woliata sodo, Ethiopia

Full list of author information is available at the end of the article
} 


\section{Background}

The demand for the whole blood transfusion is rising in relation to increased life expectancy, accidents, severe anemia, cancer, chronic diseases, pregnancy-related complication and technological advancements in the healthcare delivery system demanding blood transfusion [1-3].

Among the different types of blood donors, the safest blood comes from voluntary blood donors. In different countries, the largest proportion of hospital obtained blood is replacement donation [4]. The World Health Organization (WHO) policy is to achieve $100 \%$ not-paid blood donation practice in 2020 [2].

There is a constant effort made to increase voluntary blood donation practice. Voluntary unpaid donors are the safest group who gives blood regularly [5]. Only $60 \%$ of the people in developing countries have adequate knowledge towards blood donation. The blood donation rate in low-income, middle-income, and high-income countries is $3.9,36.8$ and 11.7 per 1000 population, respectively $[6,7]$.

Willingness to donate blood without expecting financial reward is one major factor to influence blood donation practice. Donor eligibility, negative attitude and lack of education lead to blood shortage in various facilities [8]. Worldwide blood donation practices are increasing day by day, yet it is a big concern for many countries. Safe blood prevents blood borne infections from the donor to the recipient. Furthermore, it saves millions of lives each year. Blood donation is included as the main aspect of the preventive and therapeutic component of the health care delivery system [9]. Blood is a scarce product. There is an imbalance between the demand and supply [2]. In developing countries, the community accessed around $40 \%$ of the blood banks supply and from this, $60 \%$ are collected from paid blood donation [10]. This is associated with the hesitation to donate blood. People assume that they may develop complication from donating. This is a major misconception underlying the practice [11].

There is a need to establish a global and a nationwide organization in initiating and leading the practice of blood donation. Red Cross and Red Crescent society are institutions working in developing countries to address the blood donation for the mothers delivering, for patients undergoing surgery and for the patients who suffered from accidents [12].

Bleeding might be caused by accidents, medical procedures and giving birth.495,000 women die from bleeding associated with pregnancy and childbirth, which needs early medical interventions [13]. Worldwide people from all age groups require a blood donation to support continuity of life and improve the life quality [14]. This relates to the sophisticated medical surgical procedures requiring blood transfusion [15]. There is a growing need for blood. This is related to the advancement in the healthcare delivery [10]. WHO reported that $38 \%$ of voluntarily donated blood comes from those young people aged less than 25. There is a need to motivate young generations to meet $100 \%$ voluntary not-paid blood donation [16].

Young students are healthy, active, dynamic, resourceful and receptive; they constitute a greater proportion of the population so that those young students need encouragement and motivation to donate blood voluntarily. Absence of volunteer blood donation is a main challenge seen in our study area. Furthermore, no previous comparative study conducted in this area, this study tried to come up with the following major finding that fills the existing information gap on the level of knowledge, attitude and practice towards voluntary blood donation by comparing Health Science and Non-Health Science students of both universities.

\section{Methods \\ Study area and period}

The study was conducted in Adama Science and Technology University (ASTU) and Arsi university regular undergraduate Non-Health Science and Health Science students respectively. ASTU is found in Adama town East Shewa zone in Oromiya regional state. It is $100 \mathrm{~km}$ far from the capital city of Ethiopia, Addis Ababa. It has 9737 regular undergraduate students 7205 male \& 2512 Female students among them 7448 are 3rd year and above. Arsi University is found in Oromiya regional state, Arsi zone, in Aselatown that is around $170 \mathrm{~km}$ far from Addis Ababa. There are around 1130 regular undergraduate Health Science students in this university.764 of them are male and 333 are female students. Among them, 538 are from 3rd year up to 5 th year. The study was conducted from April 11-May 2, 2016.

\section{Study design}

An institution based comparative cross-sectional study design was used.

\section{Population}

The Source population was all undergraduate Health Science and Non-Health Science University students and the study population was sampled students who will fulfill the inclusion criteria.

\section{Inclusion and exclusion criteria}

ASTU Non-Health Science and Arsi university Health Science regular undergraduate at least 18 years old and those students who were from third-year up to the fifthyear were included. Those students who were critically ill during the data collection time, distance program 
students, weekend students, postgraduate students, students who had chronic disease and mentally challenged students were excluded.

\section{Sample size and sampling procedure Sample size determination}

The sample size was calculated using Epi-Info version 21 by considering a $23.6 \%$ prevalence of blood donation practice from Ambo study [3], 95\% confidence level, $80 \%$ power of the study, a risk ratio of 2 , and one to one ratio (1:1)in comparison groups. After adding of 5\% Non-response rate, the final sample size became 360(in each group it became 180).

\section{Sampling procedure}

First, the two universities were selected using a stratified sampling method, then the sample was equally distributed between both universities (half of the Health Science students and the other half of Non-Health Science students).Then the sample was proportional allocated using year of study. Finally, the simple random sampling method was used.

\section{Operational definition}

Knowledge A score of one was given for the correct response and zero for wrong response. Respondents who scored above the mean scored were considered as having good knowledge and others were considered as having poor knowledge.

Attitude A score of one was given for each correct response and zero for the wrong response. Respondents who scored above the mean scored were considered as having favorable attitude and others were considered as having unfavorable attitude.

Practice It was measured by asking about the history of blood donation.

\section{Data collection tools}

Structured questionnaire The structured questionnaire was adapted after a review of different literatures $[3,11,13]$. The questionnaire had four parts. The first part questions about the socio-demographic characteristics of the respondents. The second, third and fourth part questions about the knowledge, attitude and practice level of blood donation respectively.

Data collection procedure Four data collectors were recruited. One supervisor was used during the data collection period. Training was provided for the data collectors and the supervisor for two days by the principal investigator. The sessions of the training were the objective of the study, the meaning of each question and techniques of interview. In addition, the role of the data collector and the supervisor was covered.

\section{Data quality assurance}

Training was given to supervisors and data collectors. After completing the training, a pre-test did in rift valley University College Adama campus, accounting $5 \%$ of the total sample. During the data collection period, supervisors reviewed for the completeness, consistency, and accuracy of each questionnaire. Corrective measures were taken by discussing with the research team.

\section{Data processing and analysis}

Data checked manually for completeness and then coded and entered using EpiData version 3.1.The generated data were exported to SPSS version 20. The data were cleaned by visualizing, calculating frequencies and sorting. Frequencies and proportions were computed. The statistical association was done for categorical variables. Significance was determined by using crude and adjusted odds ratios with 95\% confidence intervals. To assess the association between the dependent variables and independent variables, bivariable analysis was employed. Then multiple logistic regressions were employed to identify different predictor variables with considering $p$-value less than 0.05 . Finally, the results were presented as tables, figures and sentence.

\section{Result}

Socio-demographic characteristics

In the study, 360 students participated in the study. The median age of Health Science students was 22 with a range of 21-23 and the Non-Health Science students were 23 with a range of 22-24. Among them, 294(81.7\%) were males, 233(64.7\%) were Orthodox religion followers, 139(38.6\%) were Amhara and 199(55.3\%) came from urban areas (Table 1).

\section{Level of knowledge of blood donation}

The majority of Health Science students 143(79.4\%) of them has good knowledge regarding blood donation on the other hand only 25(13.9\%) of Non-Health Science students shown to have good knowledge generally there is a significant knowledge difference was observed (Table 2).

\section{Level of attitude on blood donation}

Less than half $84(46.7 \%)$ and $64(35.6 \%)$ of Health and Non-Health Science students have favorable attitudes towards blood donation respectively. Generally, on chisquare test, a significant difference of attitude level observed, Health Science students shown to have a better level of attitude when compared to Non-Health Science (Table 3). 
Table 1 Socio-demographic characteristics of Health Science students in Arsi University Health Science students and Adama science and Technology University Non-Health Science students, Ethiopia, April 11-May 2, 2016

\begin{tabular}{|c|c|c|c|c|c|c|c|}
\hline \multirow[t]{2}{*}{ Variables } & & \multicolumn{2}{|c|}{ Non-Health Science student } & \multicolumn{2}{|c|}{ Health Science student } & \multicolumn{2}{|c|}{ Total $(n=360)$} \\
\hline & & $\mathrm{N}$ & $(\%)$ & $\mathrm{N}$ & $(\%)$ & $\mathrm{N}$ & $\%$ \\
\hline \multirow[t]{2}{*}{ Age } & $18-24$ & 152 & 84.4 & 152 & 84.4 & 304 & 84.4 \\
\hline & $25-30$ & 28 & 15.6 & 28 & 15.6 & 56 & 15.6 \\
\hline \multirow[t]{2}{*}{ Gender } & Male & 152 & 84.4 & 142 & 78.9 & 294 & 81.7 \\
\hline & Female & 28 & 15.6 & 38 & 21.1 & 66 & 18.3 \\
\hline \multirow[t]{3}{*}{ Religion } & Orthodox & 117 & 65 & 116 & 64.4 & 233 & 64.7 \\
\hline & Protestant & 34 & 18.9 & 34 & 18.9 & 68 & 18.9 \\
\hline & Muslim & 29 & 16.1 & 30 & 16.7 & 59 & 16.4 \\
\hline \multirow[t]{3}{*}{ Ethnicity } & Amhara & 76 & 42.2 & 63 & 35 & 139 & 38.6 \\
\hline & Oromo & 48 & 26.7 & 90 & 50 & 138 & 38.3 \\
\hline & Others & 56 & 31.1 & 27 & 15 & 83 & 23.1 \\
\hline \multirow[t]{2}{*}{ Original Residence } & Rural & 74 & 41.1 & 87 & 48.3 & 161 & 44.7 \\
\hline & Urban & 106 & 58.9 & 93 & 51.7 & 199 & 55.3 \\
\hline \multirow[t]{3}{*}{ year of study } & Third year & 90 & 50 & 72 & 40 & 162 & 45 \\
\hline & Fourth Year & 50 & 27.8 & 65 & 36.1 & 115 & 31.9 \\
\hline & Fifth year & 40 & 22.2 & 43 & 23.9 & 83 & 23.1 \\
\hline
\end{tabular}

Level of practice of blood donation

Pertaining to blood donation practices 49 (27.2\%) and 41 (22.8\%) of Health and Non-Health Science students donate blood at least once in their lifetime respectively. A significant difference was observed regarding the level of blood donation practice between health and NonHealth Science students (Table 4).

\section{Factors associated with the level of knowledge among} health science students

Multivariable logistic regression was used to identify factors associated with the level of knowledge about blood donation. Consequently, coefficients were expressed as crude and adjusted OR relative to the referent category. The gender of the students was found as the significant predictor. Accordingly, female Health Science students were 3.2 times more likely to have a better knowledge than male Health Science students were $(\mathrm{AOR}=3.150$, 95\% CI: 1.313, 7.554) (Table 5).
Factors associated with level of attitude among health science students

Multivariable logistic regression was used to identify factors associated with attitudes about blood donation. The ethnicity of the students was found as the significant predictor. Health Science students from the Oromo ethnic group were 2.1 times more likely to have a favorable attitude as compared to Health Science students from the Amhara ethnic group $(\mathrm{AOR}=2.085,95 \% \mathrm{CI}$ : 1.025 , 4.243) (Table 6).

\section{Factors associated with level of practice among health science students}

Multivariable logistic regression was used to identify factors associated with practice of blood donation. The gender of the students was found as the significant predictor. Accordingly, Female Health Science students were 65.7\% less likely to donate blood than male Health Science students were $(\mathrm{AOR}=0.343,95 \% \mathrm{CI}: 0.151,0.779)$ (Table 7).

Table 2 Level of knowledge of blood donation among Health Science students in Arsi University and Non-Health Science students in Adama Science and Technology University, Ethiopia, April 11-May 2, 2016

\begin{tabular}{|c|c|c|c|c|c|c|}
\hline \multirow[t]{2}{*}{ Variables } & \multicolumn{2}{|c|}{ Health Science students } & \multicolumn{2}{|c|}{ Non-Health Science students } & \multirow[t]{2}{*}{$x^{2}$} & \multirow[t]{2}{*}{$P$ value } \\
\hline & $\mathrm{n}$ & $\%$ & $\mathrm{n}$ & $\%$ & & \\
\hline \multicolumn{7}{|c|}{ Level of knowledge } \\
\hline Good & 143 & 79.4 & 25 & 13.9 & \multirow[t]{2}{*}{152.779} & \multirow[t]{2}{*}{$<0.001^{\mathrm{a}}$} \\
\hline Poor & 37 & 20.6 & 155 & 86.1 & & \\
\hline
\end{tabular}


Table 3 Level of attitude on blood donation among Health Science students in Arsi University and Non-Health Science students in Adama Science and Technology University, Ethiopia, April 11-May 2, 2016

\begin{tabular}{|c|c|c|c|c|c|c|}
\hline \multirow[t]{2}{*}{ Variables } & \multicolumn{2}{|c|}{ Health Science students } & \multicolumn{2}{|c|}{ Non-Health Science students } & \multirow[t]{2}{*}{$x^{2}$} & \multirow{2}{*}{$\begin{array}{l}P \\
\text { value }\end{array}$} \\
\hline & $\mathrm{n}$ & $\%$ & $n$ & $\%$ & & \\
\hline \multicolumn{7}{|l|}{ Level of Attitude } \\
\hline Favorable & 84 & 46.7 & 64 & 35.6 & 4.142 & $0.042^{\mathrm{a}}$ \\
\hline Non-favorable & 96 & 53.3 & 116 & 64.4 & & \\
\hline
\end{tabular}

${ }^{\text {a }}$ Significant association

Factors associated with level of knowledge among nonhealth science students

Multivariable logistic regression used to identify factors associated with the level of knowledge about blood donation. Before adjustment, Fifth-year Non-Health Science students were $66.7 \%$ less likely to have higher knowledge than third-year Non-Health Science (AOR = 0.333, 95\% CI: 0.123, 0.900).However, it was not any more significant after adjustment.

After adjustment, Religion and residence of the students were significant predictors. Accordingly, Protestant religion follower Non-Health Science students were 10.2 times more likely to have a better knowledge than Orthodox religion follower Non- Health Science students $(A O R=10.173$, 95\% CI: 1.191,86.905). Urban living Non-Health Science students were $71.1 \%$ less likely to had good knowledge than rural living Non- Health Science students $(\mathrm{AOR}=0.289,95 \% \mathrm{CI}: 0.094,0.891)$ (Table 8).

Factors associated with the level of attitude among nonhealth science students

Multivariable logistic regression used to identify factors associated with the level of attitude about blood donation. Before adjustment, other religion follower NonHealth Science students were 3.1 times more likely to have a favorable attitude than orthodox religion follower Non-Health Science students were $(A O R=3.110$, 95\% CI: $1.108,8.732)$. Nevertheless, it was not any more significant after adjustment. Gender and year of study of the students were the significant predictor.

Accordingly, Female Non-Health Science students were $61.1 \%$ less likely to have a favorable attitude than male Non-Health Science students were $(\mathrm{AOR}=0.389$, 95\% CI: 0.152, 0.992). The fifth year Non-Health Science students were $61.1 \%$ less likely to had favorable attitude than third-year Non-Health Science $(A O R=0.389,95 \%$ CI: 0.164, 0.922)(Table 9).

\section{Factors associated with level of practice among non- health science students}

Multivariable logistic regression used to identify factors associated with the level of knowledge. Accordingly, Fourthyear Non-Health Science students were 5.2 times more likely to donate blood than third-year Non- Health Science students $(\mathrm{AOR}=5.159,95 \% \mathrm{CI}: 1.611,16.525)($ Table 10$)$.

\section{Discussion}

Maintaining the required level of blood supply is the main concern of various organizations working on health. Therefore, identifying the level of knowledge, attitude and practice is very essential. An attempt was made to identify the knowledge, attitude, practice and factors associated factors with blood donation between Health Science students of Arsi University and NonHealth Science students of Adama Science and Technology University.

The study revealed that $79.4 \%$ of Health Science students shown to have good knowledge about blood donation. This is comparable to a study conducted in Addis Ababa university Health Science students, which was (83.6\%) [17]; But this is higher when compared with the study conducted on Health Science students in Tamil Nadu, India in which around $35.6 \%$ of the respondents shown to have a good level of knowledge [18]. It is also higher than the study conducted on Health Science students of Manipur (9\%) [19]. This difference might be related to the background of the students.

Table 4 Level of practice of blood donation among Health Science students in Arsi University and Non-Health Science students at Adama Science and Technology University, Ethiopia, April 11-May 2, 2016

\begin{tabular}{|c|c|c|c|c|c|c|}
\hline \multirow[t]{2}{*}{ Variables } & \multicolumn{2}{|c|}{ Health Science students } & \multicolumn{2}{|c|}{ Non-Health Science students } & \multirow[t]{2}{*}{$x^{2}$} & \multirow{2}{*}{$\begin{array}{l}P \\
\text { value }\end{array}$} \\
\hline & $n$ & $\%$ & $n$ & $\%$ & & \\
\hline \multicolumn{7}{|c|}{ Level of practice } \\
\hline Yes & 49 & 27.2 & 41 & 22.8 & \multirow[t]{2}{*}{0.726} & \multirow[t]{2}{*}{0.394} \\
\hline No & 131 & 72.8 & 139 & 77.2 & & \\
\hline
\end{tabular}


Table 5 Factors associated with Level of knowledge on blood donation among Health Science students in Arsi University, Ethiopia, April 11-May 2, 2016

\begin{tabular}{|c|c|c|c|c|c|}
\hline \multirow[t]{2}{*}{ Variables } & & \multicolumn{2}{|c|}{ Knowledge level } & \multirow[t]{2}{*}{$\operatorname{COR}(95 \% \mathrm{Cl})$} & \multirow[t]{2}{*}{$\mathrm{AOR}(95 \% \mathrm{Cl})$} \\
\hline & & Good & Poor & & \\
\hline \multirow[t]{2}{*}{ Gender } & Male $^{a}$ & 118 & 24 & 1 & 1 \\
\hline & Female & 25 & 13 & $2.557(1.148,5.696)^{b}$ & $3.150(1.313,7.554)^{b}$ \\
\hline \multirow[t]{2}{*}{ Age } & $18-24^{a}$ & 118 & 34 & 1 & 1 \\
\hline & $25-30$ & 25 & 3 & $0.416(0.119,1.464)$ & $0.391(0.100,1.528)$ \\
\hline \multirow[t]{3}{*}{ Religion } & Orthodox ${ }^{a}$ & 91 & 25 & 1 & 1 \\
\hline & Protestant & 27 & 7 & $0.944(0.368,2.420)$ & $0.871(0.310,2.449)$ \\
\hline & Others & 25 & 5 & $0.728(0.253,2.096)$ & $0.749(0.242,2.320)$ \\
\hline \multirow[t]{3}{*}{ Ethnicity } & Amhara $^{a}$ & 48 & 15 & 1 & 1 \\
\hline & Oromo & 74 & 16 & $0.692(0.313,1.528)$ & $0.823(0.347,1.951)$ \\
\hline & Others & 21 & 6 & $0.914(0.312,2.683)$ & $0.896(0.270,2.974)$ \\
\hline \multirow[t]{3}{*}{ Year of study } & Third year ${ }^{a}$ & 59 & 13 & 1 & 1 \\
\hline & Fourth year & 47 & 18 & $1.738(0.773,3.906)$ & $2.400(0.993,5.804)$ \\
\hline & Fifth year & 37 & 6 & $0.736(0.257,2.105)$ & $0.890(0.286,2.774)$ \\
\hline \multirow[t]{2}{*}{ Residence } & Rural $^{a}$ & 69 & 18 & 1 & 1 \\
\hline & Urban & 74 & 19 & $0.984(0.477,2.029)$ & $1.027(0.465,2.266)$ \\
\hline
\end{tabular}

${ }^{\mathrm{a}}$ Reference Category, ${ }^{\mathrm{b}}$ Significant association

Table 6 Factors associated with Level of attitude on blood donation among Health Science students in Arsi University, Ethiopia, April 11-May 2, 2016

\begin{tabular}{|c|c|c|c|c|c|}
\hline \multirow[t]{2}{*}{ Variables } & & \multicolumn{2}{|c|}{ Attitude level } & \multirow[t]{2}{*}{ COR(95\% Cl) } & \multirow[t]{2}{*}{$\mathrm{AOR}(95 \% \mathrm{Cl})$} \\
\hline & & Favorable & Unfavorable & & \\
\hline \multirow[t]{2}{*}{ Gender } & Male $^{a}$ & 71 & 71 & 1 & 1 \\
\hline & Female & 13 & 25 & $1.923(0.912,4.057)$ & $1.972(0.884,4.399)$ \\
\hline \multirow[t]{2}{*}{ Age } & $18-24^{a}$ & 72 & 80 & 1 & 1 \\
\hline & $25-30$ & 12 & 16 & $1.200(0.532,2.707)$ & $1.159(0.452,2.967)$ \\
\hline \multirow[t]{3}{*}{ Religion } & Orthodox ${ }^{a}$ & 56 & 60 & 1 & 1 \\
\hline & Protestant & 18 & 16 & $0.830(0.386,1.784)$ & $0.649(0.283,1.493)$ \\
\hline & Others & 10 & 20 & $1.867(0.804,4.332)$ & $1.475(0.607,3.588)$ \\
\hline \multirow[t]{3}{*}{ Ethnicity } & Amhara $^{a}$ & 36 & 27 & 1 & 1 \\
\hline & Oromo & 36 & 54 & $2.000(1.041,3.844)^{b}$ & $2.085(1.025,4.243)^{b}$ \\
\hline & Others & 12 & 15 & $1.667(0.672,4.134)$ & $1.823(0.681,4.884)$ \\
\hline \multirow[t]{3}{*}{ Year of study } & Third year $^{a}$ & 32 & 40 & 1 & 1 \\
\hline & Fourth year & 31 & 34 & $0.877(0.448,1.720)$ & $0.913(0.442,1.886)$ \\
\hline & Fifth year & 21 & 22 & $0.838(0.393,1.787)$ & $0.836(0.356,1.966)$ \\
\hline \multirow[t]{2}{*}{ Residence } & Rural $^{a}$ & 43 & 44 & 1 & 1 \\
\hline & Urban & 41 & 52 & $1.239(0.689,2.229)$ & $1.083(0.576,2.037)$ \\
\hline \multirow[t]{2}{*}{ Knowledge } & Good $^{a}$ & 69 & 74 & 1 & 1 \\
\hline & Poor & 15 & 22 & $1.368(0.657,2.848)$ & $1.346(0.613,2.952)$ \\
\hline
\end{tabular}


Table 7 Factors associated with Level of practice of blood donation among Health Science students in Arsi University, Ethiopia, April 11-May 2, 2016

\begin{tabular}{|c|c|c|c|c|c|}
\hline \multirow[t]{2}{*}{ Variables } & & \multicolumn{2}{|c|}{ Practice level } & \multirow[t]{2}{*}{ COR(95\% Cl) } & \multirow[t]{2}{*}{$\mathrm{AOR}(95 \% \mathrm{Cl})$} \\
\hline & & Yes & No & & \\
\hline \multirow[t]{2}{*}{ Gender } & Male $^{a}$ & 32 & 110 & 1 & 1 \\
\hline & Female & 17 & 21 & $0.359(0.170,0.761)^{b}$ & $0.343(0.151,0.779)^{b}$ \\
\hline \multirow[t]{2}{*}{ Age } & $18-24^{a}$ & 39 & 113 & 1 & 1 \\
\hline & $25-30$ & 10 & 18 & $0.621(0.264,1.460)$ & $0.820(0.307,2.188)$ \\
\hline \multirow[t]{3}{*}{ Religion } & Orthodox ${ }^{a}$ & 34 & 82 & 1 & 1 \\
\hline & Protestant & 8 & 26 & $1.348(0.555,3.273)$ & $1.427(0.542,3.762)$ \\
\hline & Others & 7 & 23 & $1.362(0.534,3.473)$ & $1.419(0.522,3.860)$ \\
\hline \multirow[t]{3}{*}{ Ethnicity } & Amhara $^{a}$ & 18 & 45 & 1 & 1 \\
\hline & Oromo & 24 & 66 & $1.100(0.536,2.258)$ & $0.896(0.405,1.982)$ \\
\hline & Others & 7 & 20 & $1.143(0.412,3.168)$ & $1.046(0.338,3.237)$ \\
\hline \multirow[t]{3}{*}{ Year of study } & Third year $^{a}$ & 14 & 58 & 1 & 1 \\
\hline & Fourth year & 20 & 45 & $0.543(0.247,1.192)$ & $0.461(0.195,1.091)$ \\
\hline & Fifth year & 15 & 28 & $0.451(0.191,1.061)$ & $0.466(0.177,1.229)$ \\
\hline \multirow[t]{2}{*}{ Residence } & Rural $^{a}$ & 21 & 66 & 1 & 1 \\
\hline & Urban & 28 & 65 & $0.739(0.381,1.431)$ & $0.792(0.385,1.629)$ \\
\hline \multirow[t]{2}{*}{ Knowledge } & Good $^{a}$ & 36 & 107 & 1 & 1 \\
\hline & Poor & 13 & 24 & $0.621(0.287,1.346)$ & $0.760(0.327,1.765)$ \\
\hline
\end{tabular}

${ }^{\mathrm{a}}$ Reference category, ${ }^{\mathrm{b}}$ Significant association

Table 8 Factors associated with Level of knowledge on blood donation among Non-Health Science students in Adama Science and Technology University, Ethiopia, April 11-May 2, 2016

\begin{tabular}{|c|c|c|c|c|c|}
\hline \multirow[t]{2}{*}{ Variables } & & \multicolumn{2}{|c|}{ Knowledge } & \multirow[t]{2}{*}{$\operatorname{COR}(95 \% \mathrm{Cl})$} & \multirow[t]{2}{*}{$\mathrm{AOR}(95 \% \mathrm{Cl})$} \\
\hline & & $\overline{\text { Good }}$ & $\overline{\text { Poor }}$ & & \\
\hline \multirow[t]{2}{*}{ Gender } & Male $^{a}$ & 22 & 130 & 1 & 1 \\
\hline & Female & 3 & 25 & $1.410(0.392,5.072)$ & $1.846(0.444,7.674)$ \\
\hline \multirow[t]{2}{*}{ Age } & $18-24^{\mathrm{a}}$ & 21 & 131 & 1 & 1 \\
\hline & $25-30$ & 4 & 24 & $0.962(0.303,3.051)$ & $0.861(0.228,3.247)$ \\
\hline \multirow[t]{3}{*}{ Religion } & Orthodox ${ }^{a}$ & 20 & 97 & 1 & 1 \\
\hline & Protestant & 1 & 33 & $6.804(0.879,52.687)$ & $10.173(1.191,86.905)^{b}$ \\
\hline & Others & 4 & 25 & $1.289(0.404,4.111)$ & $1.248(0.359,4.331)$ \\
\hline \multirow[t]{3}{*}{ Ethnicity } & Amhara $^{a}$ & 10 & 66 & 1 & 1 \\
\hline & Oromo & 5 & 43 & $1.303(0.417,4.075)$ & $1.171(0.345,3.973)$ \\
\hline & Others & 10 & 46 & $0.697(0.268,1.809)$ & $0.501(0.175,1.435)$ \\
\hline \multirow[t]{3}{*}{ Year of study } & Third year ${ }^{a}$ & 9 & 81 & 1 & 1 \\
\hline & Fourth year & 6 & 44 & $0.815(0.272,2.439)$ & $0.718(0.213,2.421)$ \\
\hline & Fifth year & 10 & 30 & $0.333(0.123,0.900)^{b}$ & $0.341(0.113,1.034)$ \\
\hline \multirow[t]{2}{*}{ Residence } & Rural $^{a}$ & 6 & 68 & 1 & 1 \\
\hline & Urban & 19 & 87 & $0.404(0.153,1.067)$ & $0.289(0.094,0.891)^{b}$ \\
\hline
\end{tabular}


Table 9 Factors associated with Level of attitude on blood donation among Non-Health Science students in Adama Science and Technology University, Ethiopia, April 11-May 2, 2016

\begin{tabular}{|c|c|c|c|c|c|}
\hline \multirow[t]{2}{*}{ Variables } & & \multicolumn{2}{|l|}{ Attitude } & \multirow[t]{2}{*}{$\operatorname{COR}(95 \% \mathrm{Cl})$} & \multirow[t]{2}{*}{$\mathrm{AOR}(95 \% \mathrm{Cl})$} \\
\hline & & Favorable & $\overline{\text { Unfavorable }}$ & & \\
\hline \multirow[t]{2}{*}{ Gender } & Male $^{a}$ & 50 & 102 & 1 & 1 \\
\hline & Female & 14 & 14 & $0.490(0.217,1.107)$ & $0.389(0.152,0.992)$ \\
\hline \multirow[t]{2}{*}{ Age } & $18-24^{\mathrm{a}}$ & 53 & 99 & 1 & 1 \\
\hline & $25-30$ & 11 & 17 & $0.827(0.361,1.895)$ & $0.842(0.319,2.225)$ \\
\hline \multirow[t]{3}{*}{ Religion } & Orthodox ${ }^{a}$ & 46 & 71 & 1 & 1 \\
\hline & Protestant & 13 & 21 & $1.047(0.477,2.294)$ & $0.655(0.258,1.663)$ \\
\hline & Others & 5 & 24 & $3.110(1.108,8.732)^{b}$ & $2.778(0.934,8.263)$ \\
\hline \multirow[t]{3}{*}{ Ethnicity } & Amhara $^{a}$ & 28 & 48 & 1 & 1 \\
\hline & Oromo & 18 & 30 & $0.972(0.460,2.053)$ & $0.865(0.367,2.039)$ \\
\hline & Others & 18 & 38 & $1.231(0.594,2.553)$ & $1.731(0.738,4.058)$ \\
\hline \multirow[t]{3}{*}{ Year of study } & Third year ${ }^{a}$ & 28 & 62 & 1 & 1 \\
\hline & Fourth year & 15 & 35 & $1.054(0.497,2.235)$ & $1.036(0.447,2400)$ \\
\hline & Fifth year & 21 & 19 & $0.409(0.190,0.878)^{b}$ & $0.389(0.164,0.922)^{b}$ \\
\hline \multirow[t]{2}{*}{ Residence } & Rural $^{a}$ & 25 & 49 & 1 & 1 \\
\hline & Urban & 39 & 67 & $0.877(0.470,1.634)$ & $0.914(0.448,1.868)$ \\
\hline \multirow[t]{2}{*}{ Knowledge level } & Good $^{a}$ & 12 & 13 & 1 & 1 \\
\hline & Poor & 52 & 103 & $1.828(0.780,4.289$ & $1.883(0.730,4.859)$ \\
\hline
\end{tabular}

${ }^{\mathrm{a}}$ Reference category, ${ }^{\mathrm{b}}$ Significant association

Table 10 Factors associated with Level of practice of blood donation among Non-Health Science students in Adama Science and Technology University, Ethiopia, April 11-May 2, 2016

\begin{tabular}{|c|c|c|c|c|c|}
\hline \multirow[t]{2}{*}{ Variables } & & \multicolumn{2}{|c|}{ Practice level } & \multirow[t]{2}{*}{ COR(95\% Cl) } & \multirow[t]{2}{*}{ AOR(95\% Cl) } \\
\hline & & Yes & No & & \\
\hline \multirow[t]{2}{*}{ Gender } & Male $^{a}$ & 35 & 117 & 1 & 1 \\
\hline & Female & 6 & 22 & $1.097(0.412,2.918)$ & $1.830(0.619,5.411)$ \\
\hline \multirow[t]{2}{*}{ Age } & $18-24^{a}$ & 75 & 229 & 1 & 1 \\
\hline & $25-30$ & 15 & 41 & $1.428(0.506,4.026)$ & $1.476(0.456,4.782)$ \\
\hline \multirow[t]{3}{*}{ Religion } & Orthodox ${ }^{a}$ & 64 & 169 & 1 & 1 \\
\hline & Protestant & 12 & 56 & $2.586(0.841,7.948)$ & $2.093(0.592,7.397)$ \\
\hline & Others & 14 & 45 & $1.084(0.421,2.792)$ & $1.184(0.422,3.326)$ \\
\hline \multirow[t]{3}{*}{ Ethnicity } & Amhara $^{a}$ & 38 & 101 & 1 & 1 \\
\hline & Oromo & 32 & 106 & $1.786(0.715,4.458)$ & $1.332(0.494,3.591)$ \\
\hline & Others & 20 & 63 & $1.181(0.529,2.638)$ & $0.932(0.375,2.316)$ \\
\hline \multirow[t]{3}{*}{ Year of study } & Third year ${ }^{a}$ & 41 & 121 & 1 & 1 \\
\hline & Fourth year & 24 & 91 & $4.929(1.613,15.056)^{b}$ & $5.159(1.611,16.525)$ \\
\hline & Fifth year & 25 & 58 & $1.286(0.552,2.996)$ & $1.592(0.620,4.090)$ \\
\hline \multirow[t]{2}{*}{ Residence } & Rural $^{a}$ & 37 & 124 & 1 & 1 \\
\hline & Urban & 53 & 146 & $0.894(0.438,1.822)$ & $0.985(0.430,2.255)$ \\
\hline \multirow[t]{2}{*}{ Knowledge level } & Good $^{a}$ & 45 & 123 & 1 & 1 \\
\hline & Poor & 45 & 147 & $2.162(0.875,5.342)$ & $2.064(0.747,5.701)$ \\
\hline
\end{tabular}


The study also revealed that less than half $(46.7 \%)$ of Health Science students had a favorable attitude. This finding is different from the finding of a study conducted in South Indian in which $87.3 \%$ of the respondents show favorable attitude [20]; it is also lower than the study conducted on Addis Ababa University health-science students of Ethiopia, in which $68 \%$ of the respondents had a favorable attitude [17]. This difference might occur due to sociocultural difference and educational attributes between the respondents. This suggests the need for more emphasis on blood donation in the Health Science curriculum.

A significant good level of knowledge difference observed between Health Science students (79.4\%) and Non-Health Science students (13.9\%). In addition, significant favorable attitude differences observed between Health Science students (46.7\%) and Non-Health Science students (35.6\%). This might be due to the effect of education delivered for Health Science students. In addition, direct exposure in the hospital environment might improve the knowledge and attitude of Health Science students.

Unlike previous reports year of study was not a significant association with knowledge towards blood donation among Health Science students [17, 21]. This might be due to the absence of special education related to blood donation delivered to senior students.

In the study, female Non-Health Science students were 61.1\% less likely to have a favorable attitude than male Non-Health Science students did. This might be due to the less frequent mass-media exposure of females. This in turn might decrease access to information provided on blood donation. In addition, it might be due to the cultural taboo. This implies there is a need for health information delivery through campaigns and different sources of information.

Female Health Science students were $65.7 \%$ less likely to donate blood than male Health Science students were. This finding is consistent with similar studies [22-24]. This might be related to the limited opportunity and freedom of choice offered for females. This implies that females need to be advised more on the medical benefits of donating blood.

27.2\% Health Science students have donated blood. This is contrary to a study conducted in South India in which $10.75 \%$ have donated blood [18], in Canada $43.8 \%$ have donated blood [25]. This might be due to the difference in the implementation of blood donation campaigns. In addition, the rate of blood donation in developing country is low [26]. This implies the health policy designed in those countries need to give due emphasis to blood donation practice. Furthermore, making Blood Banks available in different health institutions might strengthen the involvement of the institutions in promoting the practice.
Knowledge has no significant association with the level of practice among Health Science students. This is similar to different studies $[27,28]$. This might be due to the lack of opportunity and have not asked for donating blood by donor recruitment programs. To increase the number of volunteer blood donors, students need to be constantly encouraged to donate blood through different blood campaigns.

The limitation of this study was the response might have been liable to social desirability bias. The study design cannot assess the cause and effect relationship. In addition, the factors expected to influence knowledge, attitude and practice may not be exhaustive. There could be other factors, which the study did not reveal. It has to be noted that the finding of this study mainly reflects the situation in Adama Science and Technology University and Arsi University. Therefore, the findings should be interpreted with caution.

The result signifies that the universities should establish or res-strengthen blood donation clubs. The students should receive training on voluntary blood donation. Furthermore, both universities need to work with various agencies to remove the misconceptions. Students also need to be motivated with recognitions. The national health policies need to focus on blood donation campaigns through the mobilization of students.

\section{Conclusion}

A significant level of knowledge difference and level of attitude difference observed between the Health Science of Arsi University and Non-Health Science students of Adama science and Technology University. There was no difference in the practice of blood donation between the two groups. The gender of the students was a significant predictor of the level of knowledge and level of practice of Health Science students. The ethnicity of the students was a significant predictor of the level of an attitude of Health Science students. Religion and residence of the students' was the significant predictor of the level of knowledge of Non-Health Science students. Gender and year of study of the students were significant predictors of the level of an attitude of Non-Health Science students. The year of a study of the students was a significant predictor of the level of practice of Non-Health Science students.

\section{Abbreviations}

AOR: Adjusted Odds Ration; ASTU: Adama Science and Technology University; Cl: Confidence Interval; COR: Crude Odds Ration; WHO: World Health Organization

\section{Acknowledgements}

We would like to forward our deepest appreciation to the Adama General Hospital and Medical College for their cooperation on necessary materials and supports to undertake this study. Finally, our appreciation also goes to the data collectors, supervisors and students who participated in the study. 


\section{Funding}

Adama Hospital and Medical College have covered the required funds for the research project. The funding body has no contribution in other activities of the research.

\section{Availability of data and materials}

The spreadsheet data supporting the finding of this thesis is available at the hands of the corresponding author. It can be delivered to the journal based on request at any time.

\section{Authors' contributions}

HWG conceptualized and designed the study. HWG, ROF and SGA analyzed, interpreted the data, drafted the manuscript and critically reviewed the manuscript. All the authors read and approved the manuscript.

\section{Ethics approval and consent to participate}

Ethical clearance letter obtained from Research and Ethics Committee of Adama Hospital and Medical College. The Ethics Committee approved obtaining the verbal consent. Additionally an informed verbal consent obtained from each respondent after providing sufficient information for the purpose of study and the right to refuse participation or to jump some questions unwilling to answer. To ensure the confidentiality, name of respondents' was not written on the questionnaires.

\section{Consent for publication}

Not applicable.

\section{Competing interests}

The authors declare that they have no competing interests.

\section{Publisher's Note}

Springer Nature remains neutral with regard to jurisdictional claims in published maps and institutional affiliations.

\section{Author details}

'Department of public Health, College of Health Sciences, Adama General Hospital and Medical College, Adama, Ethiopia. ${ }^{2}$ Department of Nursing, College of Health Sciences and Medicine, Woliata Sodo University, PO-Box: 138, Woliata sodo, Ethiopia. ${ }^{3}$ Department of Social and Population Health, Adama General Hospital and Medical College, Adama, Ethiopia.

Received: 14 February 2017 Accepted: 3 November 2017 Published online: 21 November 2017

\section{References}

1. Mohammed H, Osman T. Voluntary Blood Donation among Medical Students in a Resource-limited Country. JPHDC. 2016;2(3):257-67.

2. World Health Organization. Voluntary unpaid blood donations must increase rapidly to meet 2020 goal. http://www.who.int/campaigns/worldblood-donor-day/2016/event/en/. Accessed 17 Mar 2016.

3. Nigatu A, Demissie DB. Knowledge, Attitude and Practice on Voluntary Blood Donation and Associated Factors among Ambo University Regular Students, Ambo Town, Ethiopia. JCMHE. 2014;4(5). doi:10.4172/2161-0711.1000315.

4. Suchetha S, Muninarayana C, Venkatesha M, Anil NS. Blood donation awareness and beliefs among medical and nursing students. Int J Med Sci Public Health. 2015;4(10):1338-42

5. Tapko JB, Toure B, Sambo LG. Status of blood safety in the WHO African region: report of the 2010 survey. Brazzaville: WHO Regional Office for Africa; 2014

6. Riley W, Schwei M, McCullough J. The United States potential blood donor pool: estimating the prevalence of donor-exclusion factors on the pool of potential donors. Transfusion. 2007;47(7):1180-8.

7. World Health Organization. Blood safety and availability. WHO Fact Sheet. 2013(279). http://www.who.int/mediacentre/factsheets/fs279/en/. Accessed 17 Mar 2016.

8. Schreiber GB, Schlumpf KS, Glynn SA, Wright DJ, Tu Y, King MR, et al. Convenience, the bane of our existence, and other barriers to donating. Transfusion. 2006:46(4):545-53.

9. $\mathrm{WHO}$, "Towards $100 \%$ voluntary blood donation a global framework for action,". Melbourne, 2010. http://www.who.int/bloodsafety/publications/ 9789241599696/en/. Accessed 17 Mar 2016.
10. Mitra K, Mandal PK, Nandy S, Roy R, Joaardar GKA. Study on awareness and perceptions regarding blood safety and blood donation among health care providers in a teaching hospital of Calcutta. Indian J Community Med. 2001:26(1):21-5.

11. Zubair H, Seema HS. Comparative Study on Knowledge Attitude and Practice regarding Blood Donation in Rural and Urban area of Bangalore. Int J Med Health Sci. 2015:4(3):309-14.

12. The International Federation of Red Cross and Red Crescent Societies. Voluntary blood donation towards safe and healthy living. http://www.ifrc org/en/what-wedo/health/blood-services/global-actiontowards-100-percent-voluntary-nonremunerated-blood-donation/. Accessed 20 June 2016.

13. Hosain GM, Anisuzzaman M, Begum A. Knowledge, Attitude and practice on blood donation among Dhaka university students in Bangladesh. East Afr Med J. 1997;74(9):549-53.

14. Damesyn MA, Glynn SA, Schreiber GB, Ownby HE, Bethel J, Fridey J, et al. Behavioral and infectious disease risks in young blood donors: implications for recruitment. Transfusion. 2003:43(11):1596-603.

15. Lives S. Universal access to safe blood transfusion: scaling up the implementation of the WHO strategy for blood safety and availability for improving patient health and saving lives. Geneva: World health Organization; 2015.

16. Van Hulst M, SmitSibinga CT, Postma MJ. Health economics of blood transfusion safety-focus on sub-Saharan Africa. Biologicals. 2010;38(1):53-8.

17. Misganaw C, Tenkir M, Deresa A, Tesfaye M, Tessema TT, Taye $H$. The level and associated factors of knowledge, attitude and practice of blood donation among health science students of Addis Ababa university. IJMHSR. 2014;1(10):105-18.

18. Manikandan S, Srikumar R, Ruvanthika PN. A study on knowledge, attitude and practice on blood donation among health professional students in Chennai, Tamil Nadu, South India. IJRSP. 2013:3(3):1-4.

19. Syiemlieh AJ, Akoijam BS, Kumar S. Assessment of Knowledge, Perception \& Practice of Voluntary Blood Donation among Health Professional Students in RIMS, Imphal, Manipur. IOSR-JDMS. 2016;15(10):26-30.

20. Karakkamandapam S, Raghavan V, Sabu KM. Knowledge, attitude and practice on blood donation among health science students in a university campus. South India OJHAS. 2011;10(2):1-3.

21. Singh S, Muninarayana C, Venkatesha M, Anil NS. Blood donation awareness and beliefs among medical and nursing students. Int J Med Sci Public Health. 2015:4(10):1338-42.

22. Mamatya A, Prajapati R, Yadav R. Knowledge and practice of blood donation: a comparison between medical and non-medical Nepalese students. Nepal Med Coll J. 2012;14(4):283-6.

23. Safizadeh H, Pourdamghan N, Mohamadi B. University students Awarness and Attitude towards Blood Donation in Kerman City. IJBC. 2009;1 (3):107-10.

24. Alethea Z, Silveira MF, Dumith SC. Blood donation prevalence and associated factors in Pelotas,Southern Brazil. Rev Saude Publica. 2010:44(1):112-20.

25. Lemmens KPH, Abrham C, Ruiter RAC, Veldhuizen IJT, Bos AER, Schaalma HP. Identifying blood donors willing to help with recruitment. Vox Sang. 2008;95(3):211-7.

26. World Health organization. Global status report on road safety: time for action 2009. http://www.who.int/violence_injury_prevention/road_safety_ status/2009/en/. Accessed 5 June 2017.

27. Sabu KM, Remya A, Binu VS, Vivek R. Knowledge, Attitude and Practice on Blood Donation among Health Science students in a university campus, South India. Online J Health Allied SCS. 2011;10(2):6.

28. Bharatwaj RS, Vijaya K, Rajaram P. A Descriptive Study of Knowledge, Attitude and Practice with regard to Voluntary Blood Donation among Medical Undergraduate Students in Pondicherry, India. J Clin Diagnos Res. 2012;6(4):602-4 